\title{
Gustatory Hallucination
}

National Cancer Institute

\section{Source}

National Cancer Institute. Gustatory Hallucination. NCI Thesaurus. Code C118177.

Perception of a taste in the absence of a corresponding stimulus. 